\title{
DPY30 is required for the enhanced proliferation, motility and epithelial-mesenchymal transition of epithelial ovarian cancer cells
}

\author{
LILI ZHANG ${ }^{1,2^{*}}$, SHUGUANG ZHANG $^{2 *}$, AIHUA LI $^{2}$, ANQI ZHANG $^{2}$, SHIQIAN ZHANG $^{1}$ and LIANG CHEN ${ }^{3}$ \\ ${ }^{1}$ Department of Obstetrics and Gynecology, Qilu Hospital of Shandong University, Jinan, Shandong 250012; \\ ${ }^{2}$ Liaocheng People's Hospital, Liaocheng, Shandong 252000; ${ }^{3}$ Department of Gynecological Oncology, \\ Shandong Cancer Hospital Affiliated to Shandong University, Shandong Academy \\ of Medical Sciences, Jinan, Shandong 250117, P.R. China
}

Received March 16, 2018; Accepted August 28, 2018

DOI: $10.3892 /$ ijmm.2018.3869

\begin{abstract}
Epithelial ovarian cancer (EOC) is one of the most lethal gynecological malignancies and is known to be associated with the accumulation of various genetic and epigenetic alterations. As a member of the human histone-lysine N-methyltransferase SETD1A (SET1)/histone-lysine N-methyltransferase 2A (MLL) complexes that are required for full SET1/MLL methyltransferase activity, protein dpy-30 homolog (DPY30) catalyzes histone H3K4 methylation, and its dysfunction has been associated with the occurrence of cancer. Therefore, the present study investigated the role of DPY30 in EOC and the potential association between DPY30 expression and the clinicopathological characteristics of EOC. The expression of DPY30 was examined in EOC tissues and cell lines to identify any correlations between the clinicopathological characteristics of EOC and DPY30 expression, and to determine the effects of DPY30 on EOC cell proliferation, migration and invasion. DPY30 was highly expressed in EOC tissues and cell lines, and high DPY30 expression was significantly associated with notable clinicopathological variables in EOC patients, including International Federation of Gynecology and Obstetrics stage, pathological grade and lymph node metastasis. Functional studies on EOC cell lines demonstrated that DPY30 significantly promoted cell proliferation, migration, and invasion, accelerated cell cycle progression, and promoted epithelial-mesenchymal transition. Chromatin immunoprecipitation assay results revealed that DPY30 regulates histone $\mathrm{H} 3 \mathrm{~K} 4$ modification via interaction with the vimentin gene promoter, suggesting that DPY30
\end{abstract}

Correspondence to: Dr Shiqian Zhang, Department of Obstetrics and Gynecology, Qilu Hospital of Shandong University, 107 Wenhua West Road, Jinan, Shandong 250012, P.R. China

E-mail: zhangshiqian370112@126.com

*Contributed equally

Key words: proliferation, motility, epithelial-mesenchymal transition, epithelial ovarian cancer, histone modification, vimentin promotes the transcription of vimentin. Finally, high expression of DPY30 was significantly associated with reduced survival in patients with EOC. The results indicated that DPY30 may act as an oncogene in EOC and thus represents a potential therapeutic target and prognostic marker in EOC.

\section{Introduction}

Epithelial ovarian cancer (EOC) is the most lethal gynecological malignancy and has a number of histological subtypes (1). An advanced stage at the time of diagnosis and relapse due to chemoresistance are the principal reasons for its poor prognosis $(1,2)$, and the 5-year survival rate is only $\sim 30 \%$ (3). Therefore, there is an urgent need to elucidate the underlying mechanisms of EOC and apply the knowledge obtained to the development of novel treatments, including targeted therapy, to improve patient survival.

Various covalent modifications of histone tails, including acetylation, ubiquitination, phosphorylation and methylation, may modulate the chromatin structure and serve pivotal roles in the regulation of DNA repair, gene transcription, cell differentiation, cell cycle progression and embryonic development $(4,5)$. Histone acetylation is generally associated with transcriptional activation, and histone methylation is associated with transcriptional activation and repression. For example, methylation of histone $\mathrm{H} 3$ at the lysine 9, 20 or 27 residues (H3K9, H3K20 or H3K27, respectively) leads to transcriptional gene silencing, whereas methylation at $\mathrm{H} 3 \mathrm{~K} 4$, H3K36 or H3K79 is correlated with chromatin opening and transcriptional activation (6).

Histone H3K4 methylation is one of the most prominent epigenetic modifications associated with gene activation $(5,7)$. As the major histone $\mathrm{H} 3 \mathrm{~K} 4$ methyltransferases in mammals, histone-lysine N-methyltransferase SETD1A (SET1)/histone-lysine N-methyltransferase 2A (MLL) complexes comprise SET1, histone-lysine N-methyltransferase SETD1B, MLL, and histone-lysine N-methyltransferase 2B, $2 \mathrm{C}$ or $2 \mathrm{D}$ as the catalytic subunit, and WD repeat-containing protein 5 (WDR5), retinoblastoma-binding protein 5 (RbBp5), Set1/Ash2 histone methyltransferase complex subunit ASH2 (ASH2L) and protein dpy-30 homolog (DPY30) as integral core subunits exerting methylation activity (8-10). DPY30, a 
common member of the human SET1/MLL complexes, has been reported to be required for complete SET1/MLL methyltransferase activity $(11,12)$. DPY30 catalyzes histone H3K4 methylation, through which it regulates gene expression, cell proliferation and differentiation, and therefore affects tissue development. Furthermore, dysfunction of DPY30 may lead to the occurrence of cancer $(11,12)$.

Ovarian carcinogenesis entails the progressive accumulation of various genetic and epigenetic alterations that lead to gains of function in oncogenes and loss of function in tumor suppressor genes. Since gene transcriptional activation is affected by the chromatin structure, abnormal histone methylation, which alters the chromatin structure, is commonly associated with tumor progression and prognosis (13). Although alterations in histone methylation have been well described in various types of cancer, any alterations in histone methylation in EOC remain poorly characterized $(14,15)$. The present study aimed to examine the role of DPY30 in EOC by analyzing DPY30 expression in EOC tissues and cell lines. Correlations between the clinicopathological characteristics of EOC cases and the survival rate among patients with $\mathrm{EOC}$ were analyzed. Furthermore, the effects of DPY30 on EOC cell proliferation, migration and invasion were investigated. Finally, the mechanism of action of DPY30 was further elucidated by identifying its association with epithelial-mesenchymal transition (EMT).

\section{Materials and methods}

Clinical specimens. The present study was approved by the ethics committee of Liaocheng People's Hospital (Liaocheng, China). Written informed consent was obtained from all participants prior to surgical treatment.

Overall, 60 patients who were diagnosed with EOC and underwent cytoreductive surgery at Liaocheng People's Hospital between January 2009 and December 2011 were included in the study. The clinicopathological data of the enrolled patients were recorded. Patients were grouped by age, histological type, International Federation of Gynecology and Obstetrics (FIGO) stage, pathological grade and lymph node metastasis. The postoperative follow-up period was 5 years. An additional 20 patients with a benign ovarian epithelial tumor with a median age of 35 years (range, 18-50 years) and 15 perimenopausal patients who underwent ovariectomy due to a uterine fibroid with a median age of 54 years (range, 48-60 years) were included as controls.

Separately, 40 fresh ovarian carcinoma tissues and adjacent normal ovarian tissues were obtained from patients who underwent initial hysterectomy at Liaocheng People's Hospital between May 2014 and October 2016. All specimens were stored frozen at $-80^{\circ} \mathrm{C}$.

Cell culture. The ovarian cancer cell lines SKOV3, OVCAR3, A2780 and IOSE80 were obtained from the American Type Culture Collection (Manassas, VA, USA). SKOV3 cells were cultured in McCoy's 5A Medium (Gibco; Thermo Fisher Scientific, Inc., Waltham, MA, USA), and the OVCAR3 and A2780 lines were cultured in RPMI-1640 medium (Gibco; Thermo Fisher Scientific, Inc.). IOSE80 cells were cultured in Dulbecco's modified Eagle's medium (HyClone; GE Healthcare Life Sciences, Logan, UT, USA). All medium was supplemented with $10 \%$ fetal bovine serum (FBS; Invitrogen; Thermo Fisher Scientific, Inc.). Cells were maintained at $37^{\circ} \mathrm{C}$ with $5 \% \mathrm{CO}_{2}$ in a humidified incubator.

Immunohistochemistry. Immunohistochemical staining for DPY30 expression in EOC tissue specimens was performed as follows. The specimens were fixed in $10 \%$ neutral formalin at room temperature for $48 \mathrm{~h}$. The collected paraffin-embedded tissues were sectioned to a thickness of $4 \mu \mathrm{m}$. The slides were deparaffinized in xylene, rehydrated in graded alcohol solutions, and boiled in citrate buffer for $2.5 \mathrm{~min}$ in an autoclave. The slides were treated with $0.3 \%$ hydrogen peroxide for $10 \mathrm{~min}$ at room temperature to inhibit endogenous peroxidase activity. The slides were incubated at $4^{\circ} \mathrm{C}$ overnight with an anti-DPY30 primary antibody (cat. no. ab214010; 1:100; Abcam, Cambridge, UK). To each slide was added $100 \mu 1$ horseradish peroxidase-labeled goat anti-rabbit IgG complex (cat. no. PV-6001; OriGene Technologies, Inc., Beijing, China), which was incubated for $20 \mathrm{~min}$ at room temperature. The peroxidase reaction was developed with 3,3'-diaminobenzidine (DAB), and slides were counterstained for $2 \mathrm{~min}$ at room temperature with hematoxylin staining buffer (Sigma-Aldrich; Merck KGaA, Darmstadt, Germany). DAB was obtained from OriGene Technologies, Inc. The immunohistochemical evaluation was performed by two experienced pathologists who had no knowledge of the clinical status of the patients. Using a light microscope (BX53; Olympus Corporation, Tokyo, Japan), the digital images were processed using Image-Pro plus 6.0 software (Media Cybernetics, Inc.). Nuclear expression of DPY30 was regarded as positive. The status of DPY30 protein expression was assessed by an evaluation of the intensity of staining and the percentage of stained tumor cells.

Total RNA extraction and reverse transcription-quantitative polymerase chain reaction ( $R T-q P C R)$. Total RNA from cell lines and tissues was extracted using TRIzol ${ }^{\circledR}$ (Thermo Fisher Scientific, Inc.), according to the manufacturer's protocol. RT was performed using the PrimeScript RT Master Mix Perfect Real Time (Takara Bio, Inc., Otsu, Japan) for DPY30 (forward, 5'-ACTCGTGCCTACCTGGATCA-3' and reverse, 5'-CGA TCTTCAAACTGTGCCTTGT-3'), and GAPDH (forward, 5'-GGAGCGAGATCCCTCCAAAAT-3' and reverse, 5'-GGC TGTTGTCATACTTCTCATGG-3') was used as an internal loading control. The reaction conditions were as follows: $37^{\circ} \mathrm{C}$ for $15 \mathrm{~min}$ and $85^{\circ} \mathrm{C}$ for $5 \mathrm{sec}, 4^{\circ} \mathrm{C}$ for $10 \mathrm{~min}$. qPCR was performed using the RNA PCR kit (Takara Bio, Inc.), and SYBR-Green qPCR Master mix (Takara Bio, Inc.) was added to a $20-\mu 1$ reaction volume. Amplification was conducted using a Applied Biosystems 7500 Fast Real-Time PCR System (Thermo Fisher Scientific, Inc.) under the following conditions: Initial denaturation for $1 \mathrm{cycle}$ at $95^{\circ} \mathrm{C}$ for $30 \mathrm{sec}$, followed by denaturation at $95^{\circ} \mathrm{C}$ for $5 \mathrm{sec}$, and amplification at $60^{\circ} \mathrm{C}$ for $34 \mathrm{sec}$ for a total of 40 cycles, followed by a dissociation stage. Finally, the relative mRNA expression levels of the target genes were calculated following normalization to GAPDH mRNA expression using the $2^{-\Delta \Delta \mathrm{Cq}}$ method (16).

Stable cell line establishment by lentiviral transfection. The plasmid vector LV-pLKO-1-EGFP-puro carrying either DPY30 short hairpin (sh)RNA (LV-sh-DPY30) or 
control oligonucleotide (LV-sh-DPY30-NC) was purchased from Shanghai GenePharma Co., Ltd. (Shanghai, China). Lentiviruses were produced according to the instructions from GenePharma. The cells were infected with $30 \mu \mathrm{l}$ of each lentivirus $\left(10^{8}\right.$ particles $\left./ \mathrm{ml}\right)$ with $4 \mu \mathrm{g} / \mathrm{ml}$ polybrene (Shanghai GenePharma Co., Ltd.) for $\sim 24 \mathrm{~h}$ to establish an anti-DPY30-expressing stable cell line (SKOV3/sh-DPY30) and a control cell line (SKOV3/shDPY30-NC). The DPY30 expression levels in the established cell lines were examined by RT-qPCR using GAPDH as an endogenous control.

Protein extraction and western blotting. Total protein was extracted using radioimmunoprecipitation assay buffer (Vazyme, Piscataway, NJ, USA) with phenylmethylsulfonyl fluoride (Roche Diagnostics, Basel, Switzerland). Western blotting was performed according to the standard protocol. The concentration of protein in the supernatant was determined with a Bicinchoninic Acid Protein Assay kit (Wanleibio Co., Ltd., Shanghai, China), according to the manufacturer's protocol. A total of $30 \mu \mathrm{g}$ protein was separated by SDS-PAGE (12\% gel) and transferred to polyvinylidene difluoride membranes (EMD Millipore, Billerica, MA, USA). Following blocking with 5\% skimmed milk at room temperature for $1 \mathrm{~h}$, the membranes were incubated at $4^{\circ} \mathrm{C}$ overnight with the following primary antibodies: DPY30 (cat. no. ab214010; 1:1,000; Abcam), vimentin (cat. no. 5741; 1:1,000; Cell Signaling Technology, Inc., Danvers, MA, USA), E-cadherin (cat. no. 3195; 1:1,000; Cell Signaling Technology, Inc.), N-cadherin (cat. no. 13116; 1:1,000; Cell Signaling Technology, Inc.), zinc finger protein SNAI1 (Snail; cat. no. 3879; 1:1,000; Cell Signaling Technology, Inc.), trimethylated histone H3K4 (H3K4me3; cat. no. 9751; 1:1,000; Cell Signaling Technology, Inc.), total histone H3 (cat. no. 9728; 1:1,000; Cell Signaling Technology, Inc.) and $\beta$-actin (cat. no. T4014; 1:3,000; Abmart, Shanghai, China), which served as a loading control. Subsequently, the membranes were incubated at room temperature for $60 \mathrm{~min}$ with anti-rabbit IgG horseradish peroxidase secondary antibody (cat. no. WLA023a; 1:3,000; Wanleibio Co., Ltd.), in blocking buffer. Protein bands were visualized using an enhanced chemiluminescence system (ProteinSimple, San Jose, CA, USA) and analyzed by Quantity One software version 4.0.1 (Bio-Rad Laboratories, Inc., Hercules, CA, USA). The experiments were performed in triplicate.

\section{Functional study}

Cell proliferation. Cell proliferation was evaluated using a water-soluble tetrazolium salt assay and counted via a Cell Counting Kit-8 assay (CCK-8; Dojindo Molecular Technologies, Inc., Kumamoto, Japan). Cells (5x10³/well) were seeded on 96 -well culture plates in triplicate and incubated for 3 days at $37^{\circ} \mathrm{C}$ with $5 \% \mathrm{CO}_{2}$ in a humidified incubator. The numbers of viable cells were quantified every $24 \mathrm{~h}$ by measuring the absorbance at an optical density of $450 \mathrm{~nm}$ using a microplate reader (Epoch; BioTek Instruments, Inc., Winooski, VT, USA).

Wound-healing assay. Cells were seeded on six-well plates, and upon reaching 70-80\% confluence, the cell monolayer was scratched using a sterilized $10-\mu 1$ pipette tip. Detached cells were removed, and the plates were incubated at $37^{\circ} \mathrm{C}$ with McCoy's 5A containing 1\% FBS. Images of the scratches were capture every $24 \mathrm{~h}(0,24$ and $48 \mathrm{~h}$ total) for the assessment of cell migration. Images of at least five independent scratches were recorded, and the experiments were repeated three times.

Transwell invasion assays. Cell invasion and migration were assessed using cell culture inserts coated with or without basement membrane matrix (BD Biosciences, Franklin Lakes, NJ, USA), according to the manufacturer's protocol. A total of $\sim 5 \times 10^{5}$ cells in $100 \mu 1$ serum-free culture medium were placed in the upper chamber of triplicate wells and incubated at $37^{\circ} \mathrm{C}$ in a $5 \% \mathrm{CO}_{2}$ humidified incubator, and medium containing $20 \% \mathrm{FBS}$ was placed in the lower chamber of these wells. After $24 \mathrm{~h}$ in culture, the cells in the upper chamber were gently removed with a cotton swab, and the cells on the bottom of the insert were stained with $1 \%$ crystal violet for $20 \mathrm{~min}$ at room temperature. Cells in five random fields were counted, and the relative extents of invasion and migration were interpreted as the average cell number \pm standard deviation per field.

Cell cycle analysis. Cells $\left(1 \times 10^{6} / \mathrm{ml}\right)$ were fixed in $75 \%$ ethanol at $4^{\circ} \mathrm{C}$ overnight, washed with cold PBS, and then treated with RNaseI in a $37^{\circ} \mathrm{C}$ water bath for $30 \mathrm{~min}$, followed by propidium iodide (Wanleibio Co., Ltd.) staining for $30 \mathrm{~min}$ in darkness. Cell cycle analysis was performed via flow cytometry (BD FACSAriaII; BD Biosciences), according to the manufacturer's protocol.

Chromatin immunoprecipitation (ChIP). ChIP was performed using an EZ-Magna ChIP kit (EMD Millipore, Billerica, MA, USA), according to the manufacturer's protocol.Anti-H3K4me3 antibody (cat. no. 9751; 1:50) was obtained from Cell Signaling Technology, Inc., and anti-DPY30 antibody (cat. no. ab214010; 1:50) was obtained from Abcam. Anti-IgG (cat. no. 3900; Cell Signaling Technology, Inc.) was used as the control antibody. Vimentin promoter primers (forward, 5'-GCTGTAAGTTGG TAGCACTGA-3' and reverse, 5'-TTCTGTCGAGGGACC TAACG-3') were used in this experiment (17).

Statistical analysis. All statistical analyses were performed using SPSS version 22.0 software (IBM Corp., Armonk, NY, USA). Differences between two groups were assessed using the Student's t-test. Differences among three or more groups were evaluated using one-way analysis of variance. Data are presented as the mean \pm standard deviation from three independent experiments. Survival analysis was performed using a log-rank test and generating Kaplan-Meier plots. $\mathrm{P}<0.05$ was considered to indicate a statistically significant difference.

\section{Results}

DPY30 is upregulated in EOC tissues and cell lines. DPY30 expression in primary ovarian tissues from 95 patients was examined by immunohistochemical staining, and the associations between DPY30 expression and the clinicopathological factors of EOC were examined. The results demonstrated that the DPY30 positive staining rate was significantly increased in EOC tissues $(75 \%)$ compared with 
Table I. DPY30 expression in different ovarian tissues.

\begin{tabular}{lllr}
\hline & & \multicolumn{2}{c}{ DPY30 expression, $\mathrm{n}(\%)$} \\
\cline { 4 - 4 } Tissue type & $\mathrm{n}$ & $-(\%)$ & $+(\%)$ \\
\hline Normal ovarian tissue & 15 & $14(93.3)$ & $1(6.67)$ \\
Benign ovarian epithelial tumor & 20 & $18(90)$ & $2(10)$ \\
Epithelial ovarian cancer & 60 & $15(25)$ & $45(75)^{\mathrm{a}, \mathrm{b}}$ \\
\hline
\end{tabular}

${ }^{\text {a }} \mathrm{P}<0.05$ vs. normal; ${ }^{\text {b}} \mathrm{P}<0.05$ vs. benign. DPY30, protein dpy-30 homolog.

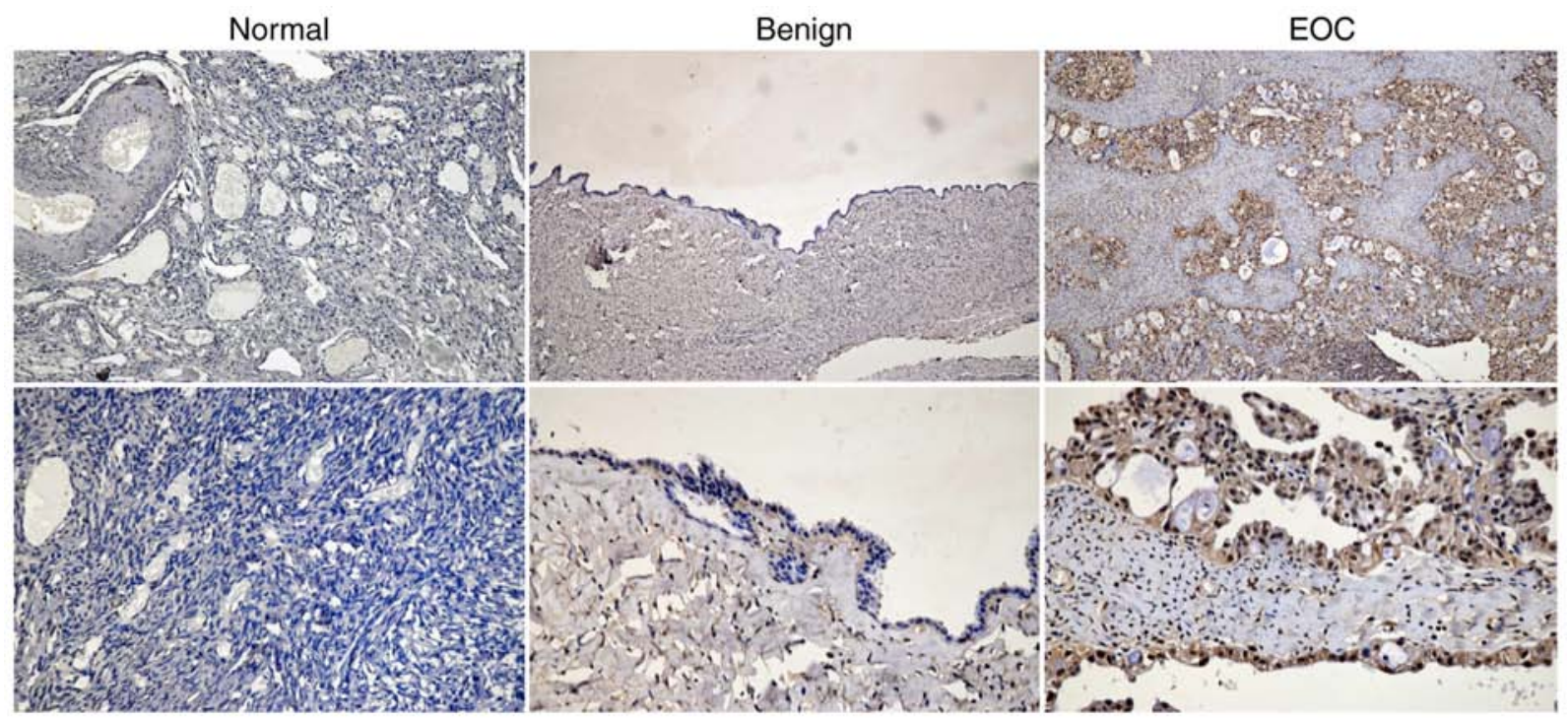

Figure 1. Immunohistochemical staining of DPY30 protein expression in normal ovarian tissues, benign ovarian epithelial tumor tissues and EOC tissues. Microscopy images illustrated a lack of staining for DPY30 expression in normal ovarian tissues, very little positive staining for DPY30 expression in benign ovarian epithelial tumors, and positive staining for DPY30 expression in EOC. Magnification: x200, upper panels; and x400, lower panels. EOC, epithelial ovarian cancer; DPY30, protein dpy-30 homolog.

benign ovarian tumors (10\%) and normal tissues $(6.67 \%$; both P<0.05; Table I; Fig. 1). Furthermore, DPY30 positive staining was observed to be associated with FIGO stage, pathological grade and lymph node metastasis in patients with EOC, although no association with histological type or age was found (Table II).

DPY30 expression was further evaluated by RT-qPCR and western blotting in $40 \mathrm{EOC}$ tissues and adjacent normal ovarian tissues as controls. Compared with normal controls, DPY30 expression at the RNA level in EOC tissues was significantly elevated (Fig. 2A). In addition, DPY30 expression at the RNA and protein levels in three EOC cell lines was higher compared with that in a normal ovarian cell line (Fig. 2B and C), and DPY30 expression was highest in SKOV3 cells among the three EOC cell lines. The upregulation of DPY30 expression in EOC suggested that it may serve an important role in EOC development.

DPY30 promotes EOC cell proliferation, migration and invasion in vitro. To further examine the role of DPY30 in the genesis and development of EOC, SKOV3 cells, which exhibited a high level of DPY30 expression (Fig. 2B and C) were transfected with LV-DPY30 shRNA to establish
SKOV3/shDPY30 stable clones (Fig. 3). The relative control clones (SKOV3/sh-DPY30-NC) were also generated. The RT-qPCR results indicated that DPY30 expression was significantly lower in SKOV3/sh-DPY30 cells compared with SKOV3/sh-DPY30-NC cells (Fig. 3A). The cell transfection efficacy was verified by RT-qPCR, and invasion and migration assays were subsequently performed.

As presented in Fig. 3B and D, SKOV3/sh-DPY30 cells exhibited diminished migratory and invasion capacities compared with SKOV3/sh-DPY30-NC cells $(\mathrm{P}<0.05)$, indicating that DPY30 expression may promote the invasion and migration of EOC cells in vitro.

According to the results for cell proliferation obtained from the CCK-8 assay, SKOV3/sh-DPY30 cells exhibited markedly inhibited proliferation compared with SKOV3/shDPY30-NC cells (Fig. 3C). Cell cycle analysis was conducted, and SKOV3/sh-DPY30 cells exhibited increased populations at the $\mathrm{G} 0 / \mathrm{G} 1$ phase and reduced populations at the $\mathrm{G} 2 / \mathrm{M}$ phase, suggesting that DPY30 expression may promote the proliferation of EOC cells in vitro (Fig. 3E).

DPY30 promotes EMT. The EMT process is critical to the acquisition of malignant traits during cancer progression (18-20). 
Table II. Correlation between DPY30 expression and the clinicopathological features of ovarian cancer.

\begin{tabular}{|c|c|c|c|c|c|}
\hline \multirow[b]{2}{*}{ Clinical pathology } & \multirow[b]{2}{*}{$\mathrm{n}$} & \multicolumn{2}{|c|}{ DPY30 expression, n (\%) } & \multirow[b]{2}{*}{$\chi^{2}$} & \multirow[b]{2}{*}{ P-value } \\
\hline & & $-(\%)$ & $+(\%)$ & & \\
\hline \multicolumn{6}{|l|}{ Age, years } \\
\hline$\leq 50$ & 14 & $4(28.6)$ & $10(71.4)$ & & \\
\hline$>50$ & 46 & $11(23.9)$ & $35(76.1)$ & 0.134 & 0.734 \\
\hline \multicolumn{6}{|l|}{ Histological type } \\
\hline Serous & 35 & $8(22.9)$ & $27(77.1)$ & & \\
\hline Endometrioid & 25 & $7(28.0)$ & $18(72.0)$ & 0.206 & 0.765 \\
\hline \multicolumn{6}{|l|}{ FIGO stage } \\
\hline I + II & 18 & $8(44.4)$ & $10(55.6)$ & & \\
\hline $\mathrm{III}+\mathrm{IV}$ & 42 & $7(16.7)$ & $35(83.3)$ & 5.185 & 0.048 \\
\hline \multicolumn{6}{|l|}{ Pathological grade } \\
\hline G1 & 11 & $6(54.5)$ & $5(45.5)$ & & \\
\hline $\mathrm{G} 2+\mathrm{G} 3$ & 49 & $9(18.4)$ & $40(81.6)$ & 6.271 & 0.021 \\
\hline \multicolumn{6}{|c|}{ Lymph node metastasis } \\
\hline Yes & 45 & $8(17.8)$ & $37(82.2)$ & 5.007 & 0.039 \\
\hline No & 15 & $7(46.7)$ & $8(53.3)$ & & \\
\hline
\end{tabular}

FIGO, International Federation of Gynecology and Obstetrics; DPY30, protein dpy-30 homolog.
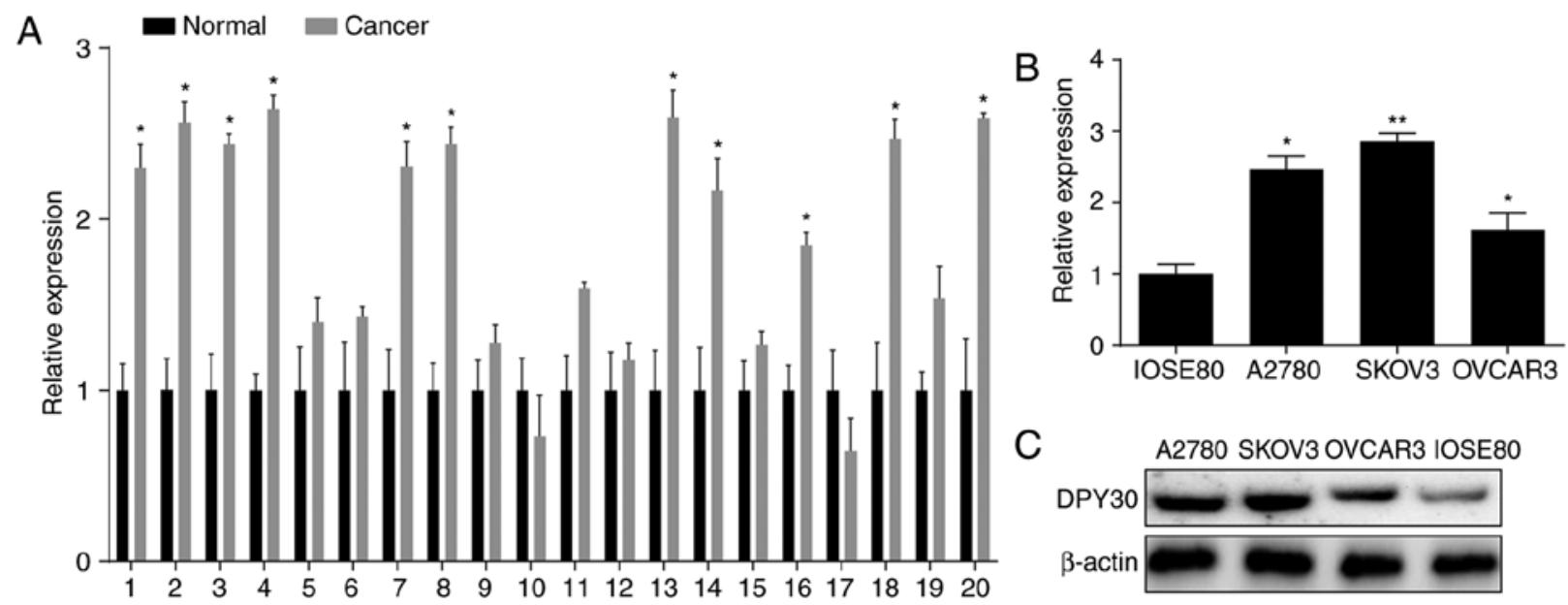

Figure 2. DPY30 is upregulated in EOC samples and cell lines. (A) Expression of DPY30 mRNA among 20 paired tumors (RT-qPCR), demonstrating increased DPY30 mRNA expression in the majority of EOC tumors compared with adjacent normal ovarian tissues. *P<0.05 vs. respective normal group. (B) DPY30 mRNA expression in three ovarian cell lines (RT-qPCR) was increased compared with IOSE80 cells. ${ }^{*} \mathrm{P}<0.05{ }^{*}{ }^{* *} \mathrm{P}<0.01 \mathrm{vs}$. IOSE80. (C) DPY30 protein expression was assessed in different ovarian cell lines compared with control IOSE80 cells (western blotting). EOC, epithelial ovarian cancer; DPY30, protein dpy-30 homolog; RT-qPCR, reverse transcription-quantitative polymerase chain reaction.

Considering the high DPY30 expression in EOC cells and its association with increased cell migration and invasion in vitro, it was hypothesized that DPY30 may be involved in the EMT process in EOC cells. The in vitro experiments demonstrated that compared with SKOV3/sh-DPY30-NC control cells, the expression of E-cadherin, an epithelial cell marker, in SKOV3/sh-DPY30 cells was significantly increased, and the expression levels of the mesenchymal cell markers vimentin, $\mathrm{N}$-cadherin and Snail were decreased (Fig. 4A). These findings supported the hypothesis that DPY30 may promote EMT in EOC cells.
DPY30 regulates vimentin expression through histone H3K4me3 modification. As mentioned above, DPY30, as a member of the human SET1/MLL complexes, is able to catalyze the methylation of histone $\mathrm{H} 3 \mathrm{~K} 4$. Methylation at $\mathrm{H} 3 \mathrm{~K} 4$, H3K36 or H3K79 is associated with chromatin opening and gene transcription activation (6), and DPY30 is primarily required for $\mathrm{H} 3 \mathrm{~K} 4 \mathrm{me} 3(8,12,21)$.

E-cadherin expression was increased upon knockdown of DPY30 expression in SKOV3 cells, whereas the expression levels of vimentin, $\mathrm{N}$-cadherin and Snail were decreased, with the greatest reduction observed for vimentin expression. In 
A

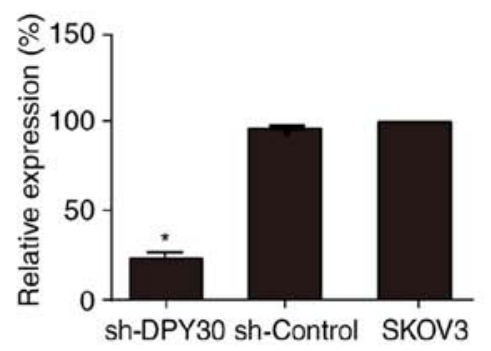

B

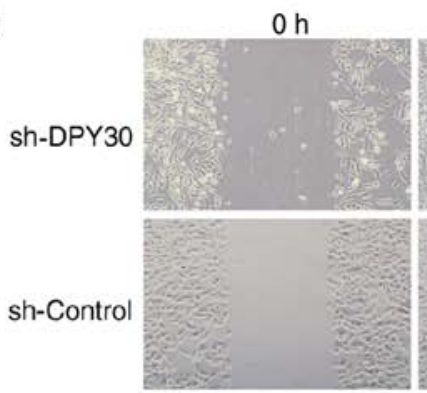

$24 \mathrm{~h}$

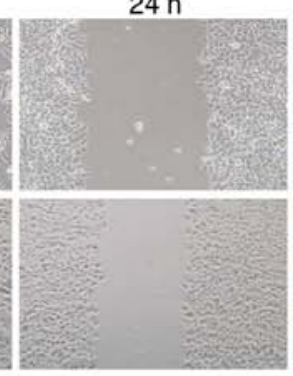

$48 \mathrm{~h}$

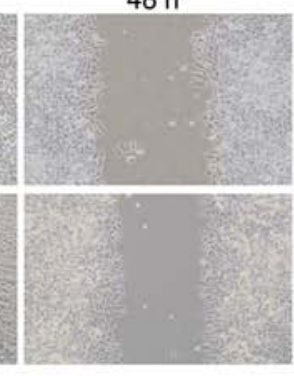

$\star \star$

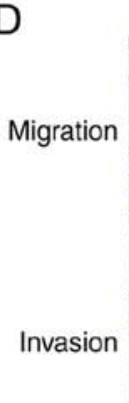

sh-DPY30 sh-Control

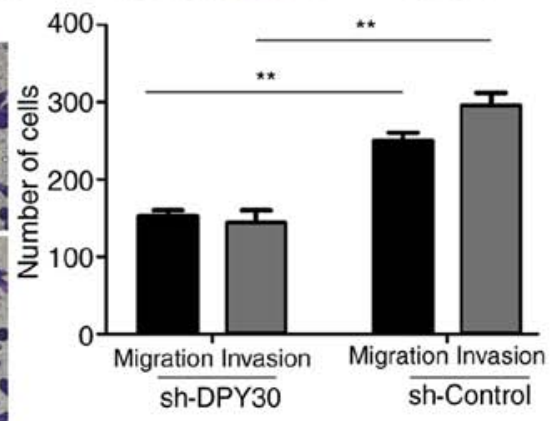

E

sh-DPY30

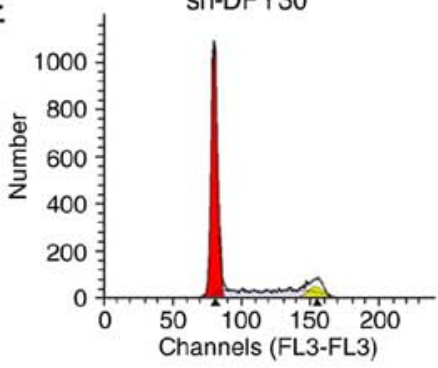

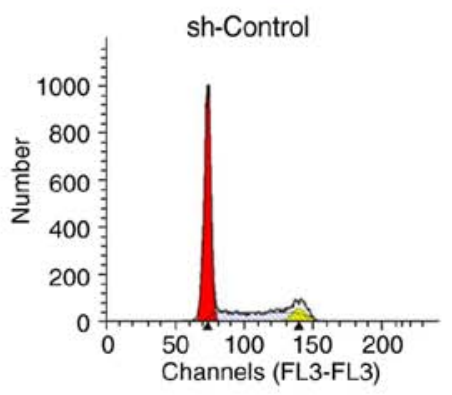
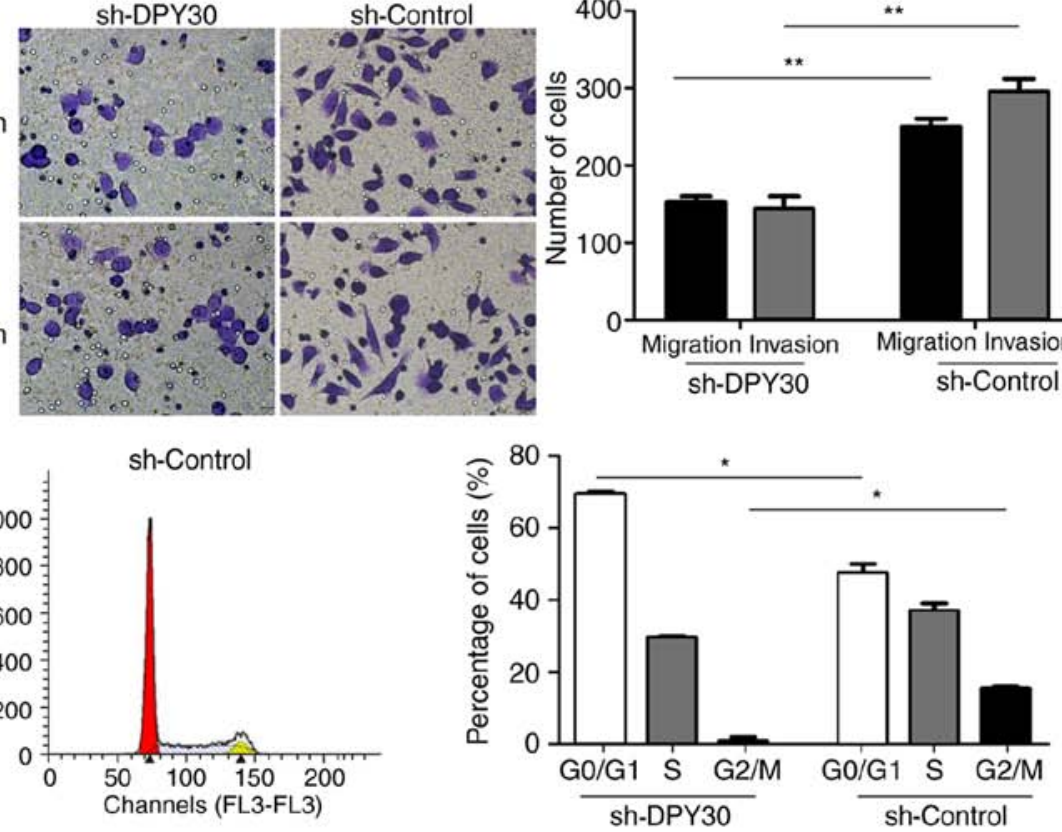

Figure 3. DPY30 promotes EOC cell proliferation, migration and invasion in vitro. (A) DPY30 expression was assessed by reverse transcription-quantitative polymerase chain reaction in SKOV3 cells transfected with the LV-DPY30 inhibitor. ${ }^{*}$ P 0.05 vs. sh-control. (B) DPY30 expression enhanced SKOV3 cell migration in a wound scratch healing assay. Magnification, x100. (C) DPY30 expression promoted SKOV3 cell proliferation. (D) DPY30 promoted SKOV3 cell migration/invasion. Magnification, x200. (E) DPY30 regulated the cell cycle, with sh-DPY30 expression in SKOV3 cells leading to a relative increase in cells at the G0/G1 phase and a concomitant decrease in cells at the G2/M phase. Data are presented as mean \pm standard deviation from three independent experiments. ${ }^{*} \mathrm{P}<0.05 ;{ }^{* *} \mathrm{P}<0.01$. sh, short hairpin; DPY30, protein dpy-30 homolog; OD, optical density.

stable SKOV3/sh-DPY30 cells, the global H3K4me3 level was upregulated upon DPY30 depletion (Fig. 4B). To investigate whether DPY30 promotes vimentin expression through H3K4me3 methylation, ChIP was performed using antibodies against DPY30 and H3K4me3, with IgG as a control. It was observed that in SKOV3/sh-DPY30 cells, the expression level of $\mathrm{H} 3 \mathrm{~K} 4 \mathrm{me} 3$ was decreased more significantly at the vimentin promoter region compared with SKOV3/shDPY30-NC control cells (Fig. 4C). These data suggested that DPY30 may regulate histone $\mathrm{H} 3 \mathrm{~K} 4$ modification at the vimentin promoter and thus enhance vimentin expression.

High DPY30 expression is associated with poor survival of patients with EOC. A survival analysis was performed in order to investigate the association between DPY30 expression levels and the survival of patients with EOC. The Kaplan-Meier method was used to estimate overall survival. From our analysis, high DPY30 expression was significantly associated with a poor prognosis in patients with EOC $(\mathrm{P}<0.05$; Fig. 5), which suggested that high DPY30 expression may affect patient survival in EOC, likely by promoting tumor metastasis.

\section{Discussion}

EOC remains a leading cause of cancer-associated mortality among women, and much research has been devoted to pursuing an effective treatment for EOC through the discovery of novel therapeutic targets. The present study focused on DPY30, a common member of the human SET1/MLL complexes that is required for complete SET1/MLL methyltransferase activity, a key process in cancer development $(11,12)$. Previously, DPY30 was reported to be essential for the differentiation and proliferation of hematopoietic progenitor cells (12) and was implicated in the differentiation potential of embryonic stem cells along the neuronal lineage (11). Research has demonstrated that depletion of DPY30 leads to a senescent-like state in cells and upregulated cyclin-dependent kinase 4 inhibitor $\mathrm{B}$ and cyclin-dependent kinase inhibitor 2A expression levels, which are directly associated with cell senescence (13). Notably, DPY30 was recently reported to be important for gastric cancer progression, suggesting that DPY30 may be a therapeutic target in gastric cancer (22).

The results of the present study indicated that DPY30 may serve important roles in EOC. The majority of ovarian cancer 

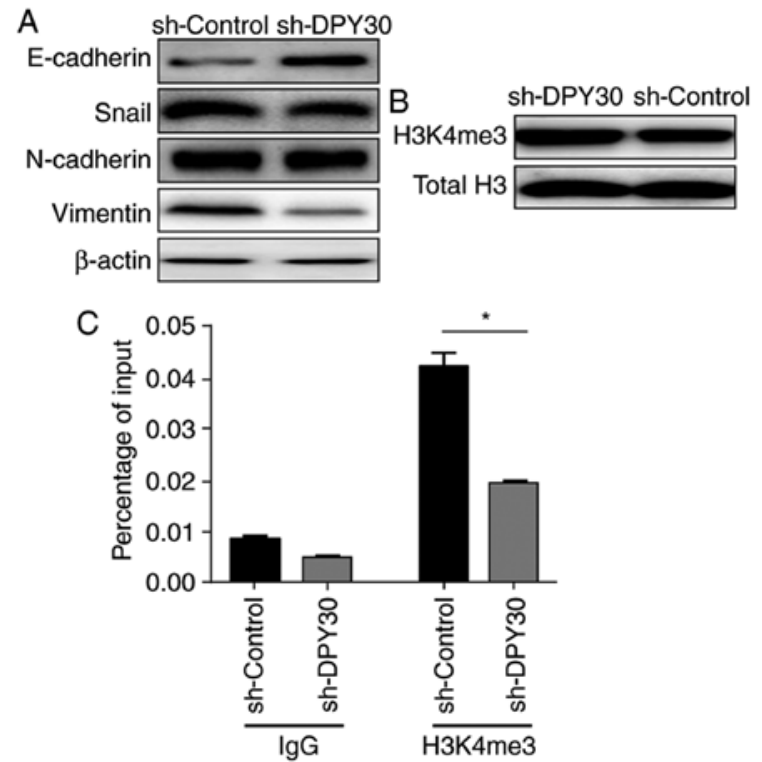

Figure 4. DPY30 expression promotes EMT in SKOV3 cells in vitro. DPY30 methylates H3K4me3 at the vimentin promoter. (A) sh-DPY30 expression enhanced the expression of the EMT marker E-cadherin and attenuated the expression of the EMT markers N-cadherin, vimentin and Snail in SKOV3 cells. (B) According to the western blotting results, H3K4me3 expression was increased in SKOV3 cells expressing sh-DPY30 compared with those expressing sh-Control. Total histone H3 served as a loading control. (C) Upon chromatin immunoprecipitation, the level of $\mathrm{H} 3 \mathrm{~K} 4 \mathrm{me} 3$ at the vimentin promoter was lower in cells expressing sh-DPY30 than in control cells. ${ }^{\text {" }} \mathrm{P}<0.05$. EMT, epithelial-mesenchymal transition; sh, short hairpin; H3K4me3, trimethylated histone H3K4; Snail, zinc finger protein SNAI1; DPY30, protein dpy-30 homolog.

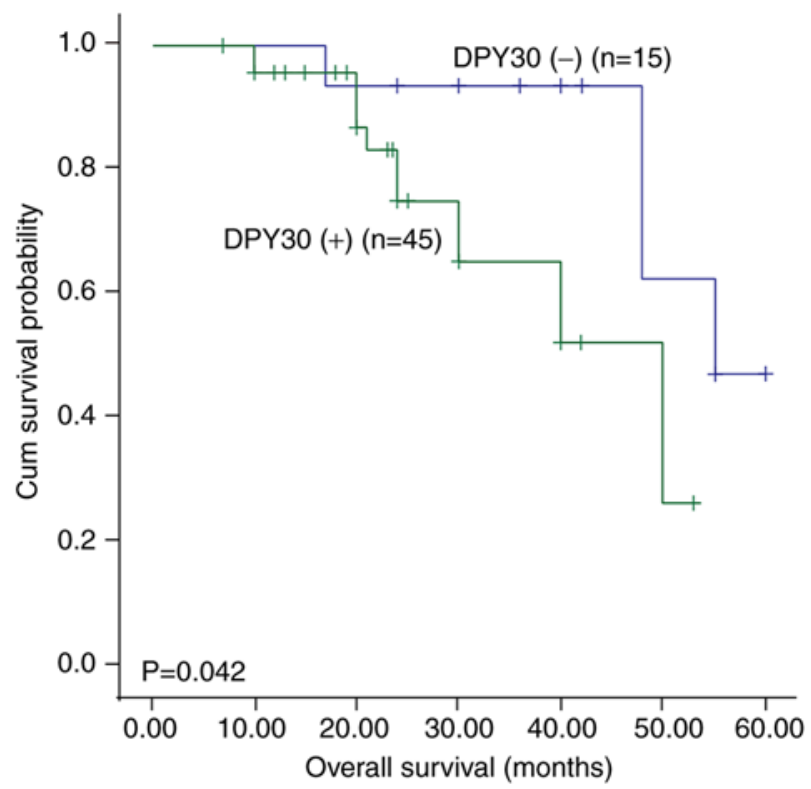

Figure 5. High DPY30 expression levels indicated shorter overall survival among Patients with epithelial ovarian cancer. DPY30, protein dpy-30 homolog; Cum, cumulative.

tissues exhibited high expression of DPY30, and DPY30 expression was positively associated with FIGO stage, pathological grade and lymph node metastasis. DPY30 expression was higher in the advanced stages (III-IV) of EOC compared with the early stages (I-II), higher in less-differentiated carci- nomas compared with well-differentiated tissues, and higher in cases with lymph node metastasis compared with those without lymph node metastasis. Therefore, the present results indicated a strong association between DPY30 and EOC development and progression.

The functional experiments further revealed that DPY30 knockdown was able to regulate the proliferation, migration and invasion of EOC cells. Importantly, DPY30 induced G0/G1 arrest in SKOV3/sh-DPY30 cells, which was further supported by the fact that DPY30 knockdown in SKOV3 cells increased the cell population at the $\mathrm{G} 0 / \mathrm{G} 1$ phase and therefore restrained cell proliferation.

The present study also indicated that DPY30 promoted EMT, a process that is important for tumor progression and metastasis $(18,23)$. DPY30 knockdown in SKOV3 cells induced increased expression of E-cadherin and decreased expression of vimentin, $\mathrm{N}$-cadherin and Snail, demonstrating a potential tumorigenic effect of DPY30 in EOC.

The underlying molecular mechanisms of the cancer-promoting effects of DPY30 have been examined in previous studies, and a number of hypotheses have been proposed (22). One hypothesis is that DPY30 overexpression leads to oncogene overexpression by increasing the methylation of histone $\mathrm{H} 3$ lysine 4 methyltransferase (H3K4MT). ASH2L or DPY30 depletion has been observed to lead to decreased H3K4me3 expression $(8,11)$. Notably, RbBp5 and WDR5 are crucial for the methylation of all three H3K4 subtypes, whereas DPY30 is primarily required for $\mathrm{H} 3 \mathrm{~K} 4 \mathrm{me} 3(5,19,21)$. In another hypothesis, overexpression of DPY30 alone increases H3K4MT methylation activity (11). Since $\mathrm{H} 3 \mathrm{~K} 4 \mathrm{me} 2 / 3$ expression is an indicator of transcriptional activity $(6,24)$, increased H3K4MT activity may directly upregulate the expression of oncogenes or downregulate the expression of tumor suppressors indirectly. Previous research found that ASH2L, another crucial component of the SET1/MLL complexes, functions as an oncoprotein $(25,26)$, which strongly supports this hypothesis. A third hypothesis is that DPY30 is able to directly activate the expression of inhibitor of DNA binding proteins via H3K4 methylation $(10,27)$. This is supported by the present finding that DPY30 promotes vimentin expression via H3K4me3 methylation at the vimentin promoter. Overall, the present study along with previous work suggested that DPY30 may promote EOC development via multiple pathways.

The results of the present study revealed important roles for DPY30 in EOC, with DPY30 acting as an oncogene and promoting EOC cell proliferation, migration and invasion capacity. The present data establish a possible mechanism through which DPY30 may promote cancer metastasis in EOC cells. DPY30 was able to promote EMT in EOC, and DPY30 promoted vimentin expression through $\mathrm{H} 3 \mathrm{~K} 4 \mathrm{me} 3$ methylation at the vimentin promoter. Therefore, DPY30 may represent a therapeutic target and prognostic marker in EOC.

\section{Acknowledgements}

The authors would like to acknowledge the continuous support and valuable guidance of Dr Haiying Chen and Dr Shaoda Ren of the Central Laboratory of Liaocheng People's Hospital (Liaocheng, China). 


\section{Funding}

The present study was supported by the Natural Science Foundation of Shandong Province (grant nos. ZR2017PH029 and ZR2015YL047) and Projects of the Medical and Health Technology Development Program in Shandong Province (grant nos. 2017WS641 and 2015WS0157).

\section{Availability of data and materials}

All data generated or analyzed during this study are included in this published article.

\section{Authors' contributions}

LZ, ShuZ and ShiZ contributed to the conception of the work and designing the study. AL and $\mathrm{LC}$ searched the literature and collated the data. LZ, LC and AZ performed the experiments. LZ analyzed the data and drafted the manuscript. ShiZ made substantial contributions to the analysis and interpretation of data, and revised the manuscript critically for important intellectual content. All authors read and approved the final manuscript.

\section{Ethics approval and consent to participate}

The present study was approved by the Ethics Committee of Liaocheng People's Hospital (Liaocheng, China). Written informed consent was obtained from all participants prior to surgical treatment.

\section{Patient consent for publication}

Consent for publication was obtained from the participants.

\section{Competing interests}

The authors declare that they have no competing interests.

\section{References}

1. Vaughan S, Coward JI, Bast RC Jr, Berchuck A, Berek JS, Brenton JD, Coukos G, Crum CC, Drapkin R, Etemadmoghadam D, et al: Rethinking ovarian cancer: Recommendations for improving outcomes. Nat Rev Cancer 11: 719-725, 2011.

2. Gao L, Ye X, Ma RQ, Cheng HY, Han HJ, Cui H, Wei LH and Chang $\mathrm{XH}$ : Low programmed cell death 5 expression is a prognostic factor in ovarian cancer. Chin Med J (Engl) 128: 1084-1090, 2015.

3. Kim MK, James J and Annunziata CM: Topotecan synergizes with CHEK1 (CHK1) inhibitor to induce apoptosis in ovarian cancer cells. BMC Cancer 15: 196, 2015.

4. Bhaumik SR, Smith E and Shilatifard A: Covalent modifications of histones during development and disease pathogenesis. Nat Struct Mol Biol 14: 1008-1016, 2007.

5. Kouzarides T: Chromatin modifications and their function. Cell 128: 693-705, 2007.

6. Berger SL: The complex language of chromatin regulation during transcription. Nature 447: 407-412, 2007.

7. Martin $C$ and Zhang Y: The diverse functions of histone lysine methylation. Nat Rev Mol Cell Biol 6: 838-849, 2005.
8. Dou Y, Milne TA, Ruthenburg AJ, Lee S, Lee JW, Verdine GL, Allis CD and Roeder RG: Regulation of MLL1 H3K4 methyltransferase activity by its core components. Nat Struct Mol Biol 13: 713-719, 2006.

9. Shilatifard A: Molecular implementation and physiological roles for histone $\mathrm{H} 3$ lysine 4 (H3K4) methylation. Curr Opin Cell Biol 20: 341-348, 2008.

10. Shilatifard A: The COMPASS family of histone H3K4 methylases: Mechanisms of regulation in development and disease pathogenesis. Annu Rev Biochem 81: 65-95, 2012.

11. Jiang H, Shukla A, Wang X, Chen WY, Bernstein BE and Roeder RG: Role for Dpy-30 in ES cell-fate specification by regulation of $\mathrm{H} 3 \mathrm{~K} 4$ methylation within bivalent domains. Cell 144: 513-525, 2011.

12. Yang Z, Augustin J, Chang C, Hu J, Shah K, Chang CW, Townes T and Jiang H: The DPY30 subunit in SET1/MLL complexes regulates the proliferation and differentiation of hematopoietic progenitor cells. Blood 124: 2025-2033, 2014.

13. Simboeck E, Gutierrez A, Cozzuto L, Beringer M, Caizzi L, Keyes WM and Di Croce L: DPY30 regulates pathways in cellular senescence through ID protein expression. EMBO J 32: 2217-2230, 2013.

14. Varier RA and Timmers HT: Histone lysine methylation and demethylation pathways in cancer. Biochim Biophys Acta 1815: 75-89, 2011.

15. Chervona $\mathrm{Y}$ and Costa M: Histone modifications and cancer: Biomarkers of prognosis? Am J Cancer Res 2: 589-597, 2012.

16. Livak KJ and Schmittgen TD: Analysis of relative gene expression data using real-time quantitative PCR and the 2(-delta delta C(T)) method. Methods 25: 402-408, 2001.

17. Liu Y, Wang Y, Chen C, Zhang J, Qian W, Dong Y, Liu Z, Zhang $X$, Wang $X$ and Zhang Z: LSD1 binds to HPV16 E7 and promotes the epithelial-mesenchymal transition in cervical cancer by demethylating histones at the Vimentin promoter. Oncotarget 8: 11329-11342, 2017.

18. Polyak K and Weinberg RA: Transitions between epithelial and mesenchymal states: Acquisition of malignant and stem cell traits. Nat Rev Cancer 9: 265-273, 2009.

19. Thiery JP: Epithelial-mesenchymal transitions in tumour progression. Nat Rev Cancer 2: 442-454, 2002.

20. Yang $J$ and Weinberg RA: Epithelial-mesenchymal transition: At the crossroads of development and tumor metastasis. Dev Cell 14: 818-829, 2008.

21. Steward MM, Lee JS, O'Donovan A, Wyatt M, Bernstein BE and Shilatifard A: Molecular regulation of H3K4 trimethylation by ASH2L, a shared subunit of MLL complexes. Nat Struct Mol Biol 13: 852-854, 2006.

22. Lee YJ, Han ME, Baek SJ, Kim SY and Oh SO: Roles of DPY30 in the proliferation and motility of gastric cancer cells. PLoS One 10: e0131863, 2015

23. Knösel T, Schlüns K, Stein U, Schwabe H, Schlag PM, Dietel M and Petersen I: Chromosomal alterations during lymphatic and liver metastasis formation of colorectal cancer. Neoplasia 6: 23-28, 2004.

24. Shi Y and Whetstine JR: Dynamic regulation of histone lysine methylation by demethylases. Mol Cell 25: 1-14, 2007.

25. South PF, Fingerman IM, Mersman DP, Du HN and Briggs SD: A conserved interaction between the SDI domain of $\mathrm{Bre} 2$ and the Dpy-30 domain of $\mathrm{Sdc1}$ is required for histone methylation and gene expression. J Biol Chem 285: 595-607, 2010.

26. Takahashi YH, Westfield GH, Oleskie AN, Trievel RC, Shilatifard A and Skiniotis G: Structural analysis of the core COMPASS family of histone H3K4 methylases from yeast to human. Proc Natl Acad Sci USA 108: 20526-20531, 2011.

27. Ohtani N, Zebedee Z, Huot TJ, Stinson JA, Sugimoto M, Ohashi Y, Sharrocks AD, Peters G and Hara E: Opposing effects of Ets and Id proteins on p16INK4a expression during cellular senescence. Nature 409: 1067-1070, 2001.

This work is licensed under a Creative Commons Attribution-NonCommercial-NoDerivatives 4.0 International (CC BY-NC-ND 4.0) License. 